Ann. Zootech., I97I, 20 (2), I85-I9I.

\title{
COMPOSITION CHIMIQUE DU LAIT DE LAPINE, ÉVOLUTION AU COURS DE LA TRAITE ET EN FONCTION DU STADE DE LACTATION
}

\author{
F. LEBAS \\ avec la collaboration technique de Marie-Claude Scheller et G. Sardi \\ Station de Recherches sur l'Elevage des Porcs, \\ Centre national de Recherches zootechniques, I. N.R. A., \\ 78 - Jouy-en-Josas

\section{RÉSUMÉ}

Divers échantillons de lait de lapine ont été obtenus à plusicurs stades successifs d'une même traite mécanique. Lors d'une traite représentant la quantité de lait consomméc normalement par les lapereaux, la composition chimique ne semble pas varier, tandis que la fraction restant dans la mamelle est plus riche en matière sèche et plus spécialement en lipides. Lors du prélèvement d'un échantillon en vue d'analyse, la quantité de lait extraite doit représenter au moins un quart de la quantité de lait présente dans la glande mammaire.

Au cours de la lactation, la composition du lait est relativement stable jusqu'à la fin de la $3^{\mathrm{e}}$ semaine. La matière sèche $(28,4 \mathrm{p}$. Ioo) est constituée principalement de protéines (49 p. Ioo) et de lipides (39 p. Ioo) tandis que lactose et cendres représentent des fractions plus faibles ( 3 p. Ioo et $8 \mathrm{p}$. Ioo respectivement). Dans le courant de la $4^{\mathrm{e}}$ semaine, le lait s'enrichit en protéines et surtout en lipides tandis que le lactose tend à disparaître.

\section{INTRODUC'TION}

Pour le Lapereau, comme pour les autres jeunes mammifères, le lait maternel constitue le seul aliment du tout jeune âge. La croissance est fonction de la quantité de lait ingérée et de sa qualité. Au cours de travaux antérieurs, nous avons décrit les variations des quantités consommées (LEBAS, I969). A l'occasion de travaux sur l'évolution de l'équipement enzymatique du pancréas exocrine (LEBAs et al., I97I), nous avons été amenés à contrôler la composition de l'ingéré des lapereaux et du lait en particulier. Ces résultats étant obtenus sur des lapins de race Californienne, il nous a semblé intéressant de les rapporter ici afin de pouvoir les comparer à ceux 
décrits par d'autres auteurs comme CoATEs et al. (rg64) et CowIE (I969) avec d'autres races. Une étude préliminaire nous a permis de déterminer la composition du lait au cours d'une même traite. Cette opération nous a semblé nécessaire pour préciser la quantité minimum de lait à prélever pour avoir un échantillon de lait réellement représentatif de ce que consomment les lapereaux.

\section{MA'TÉRIEL, E'T MÉTHODES}

\section{Animaux}

Ln ensemble de dix lapines de race Californienne a été utilisé pour la présente étude. Elles ont été élevées dans les conditions standard de notre élevage (LEBAS, I968), les lapines étant totalement séparées de leurs portées en dehors des tétées.

Quatre ont été traites une seule fois le $2 \mathrm{I}^{\mathrm{e}}$ jour après la mise bas en vue de déterminer la composition du lait ingéré par les lapereaux du début à la fin de la tétée. Six autres lapines ont été traites 9 fois à divers intervalles au cours de la période d'allaitement (o-3o jours). Les portées de ces différentes lapines étaient toutes égalisées à 8 lapereaux.

\section{Prélèvements}

Les divers échantillons de lait ont été récoltés mécaniquement grâce à la machine à traire que nous avons déjà décrite (LEBAS, 1970). Il nous semble utile cependant de rappeler que nous pratiquons une injection de I UI d'ocytocine sous forme de posthypophyse. Pour les 4 premières lapines, la quantité totale à extraire a été déterminée en fonction de la moyenne des quantités de lait réellement consommées les 2 jours précédents par les lapereaux. Au cours de la traite nous avons changé 3 fois le flacon de réception du lait de manière à obtenir 4 fractions sensiblement égales pour un même prélèvement (tabl. I). Chaque changement de flacon a nécessité une interruption de traite de ro secondes maximum.

\section{TABLEAU I}

Importance des quantités de lait prélevées pour chaque fraction d'une même traite mécanique chez 4 lapines

\begin{tabular}{|c|c|c|c|c|c|c|}
\hline \multirow{2}{*}{ No lapine } & \multicolumn{4}{|c|}{$\begin{array}{l}\text { Quantité prélevée } \\
\text { pour chaque fraction }(g)\end{array}$} & \multirow{2}{*}{$\begin{array}{c}\text { Total } \\
\text { (g) }\end{array}$} & \multirow{2}{*}{$\begin{array}{l}\text { Moyenne des } \\
2 \text { tétées des } \\
\text { lapereaux (g) }\end{array}$} \\
\hline & I & II & III & IV & & \\
\hline 1 & 65 & 46 & 47 & 57 & 215 & 237 \\
\hline 2 & 46 & 57 & 48 & 46 & 197 & 205 \\
\hline 3 & 48 & 59 & $6 t^{\prime}$ & $5:$ & 223 & 220 \\
\hline 4 & 59 & 63 & 70 & 68 & 260 & 253 \\
\hline
\end{tabular}

Pour les six autres lapines, des échantillons de 40 à $80 \mathrm{~g}$ seulement ont été prélevés sauf le I er jour après la mise bas où le prélèvement n'a pas dépassé Io $g$ pour chaque lapine. En effet, les lapines devaient en plus élever normalement leurs portées; il ne pouvait donc être question de récolter la totalité du lait présent. Les échantillonnages ont été réalisés I, 7, 9, I I, I 4, I 7, 2 I, 24 et $3^{\circ}$ jours après la mise-bas sur chacune des 6 lapines. Ces stades ont été fixés en fonction de l'équipement enzymatique du pancréas des lapereaux et non de la lactation elle-même. Ils couvrent cependant l'ensemble de la période d'allaitement avec un écart maximum de 6 jours. 
Analyses

Les divers échantillons de lait ont été analysés individuallement sauf pour la récolte le I er jour après la mise bas où un échantillon moyen a été saul analysé. Sur chácun a été céterminé la teneur en matières sèches ( $60^{\circ} \mathrm{C}$ sous vidœ), en cendres ( 12 həures à $55^{\circ} \mathrm{C}$ ), en protéines totales ( $\mathrm{N}$ Kjeldhal $\times 6,3^{8}$ ) et en lactose (méthode de Somogy I). Les matières grasses ont été cstimées par différence.

\section{RÉSULTATS}

\section{I. - Evolution de la composition du lait au cours de la traite}

L'importance des quantités prélevées a été donnée au tableau I. Sur l'ensemble, la quantité totale du lait prélevée peut être estimée comparable à la fraction du lait présent, effectivement consommée par les lapereaux. Dans tous les cas, nous avons cessé volontairement de traire à la fin de la $4^{\text {e }}$ fraction, nous n'avons jamais cherché à obtenir plus que ce qu'auraient tété les lapereaux. Il ne s'agit pas d'une traite complète et totale de la glande mammaire mais d'un simple échantillonnage.

La composition chimique moyenne des quatre fractions, rapportée au tableau 2 laisse penser que les 4 échantillons successifs ont une composition comparable. L,es tests statistiques, dans la mesure où l'on peut les appliquer à 4 mesures, ne permettent de déceler aucune différence significative.

\section{TABLEAU 2}

Composition chimique des 4 fractions constituant une traite complete de la lapine

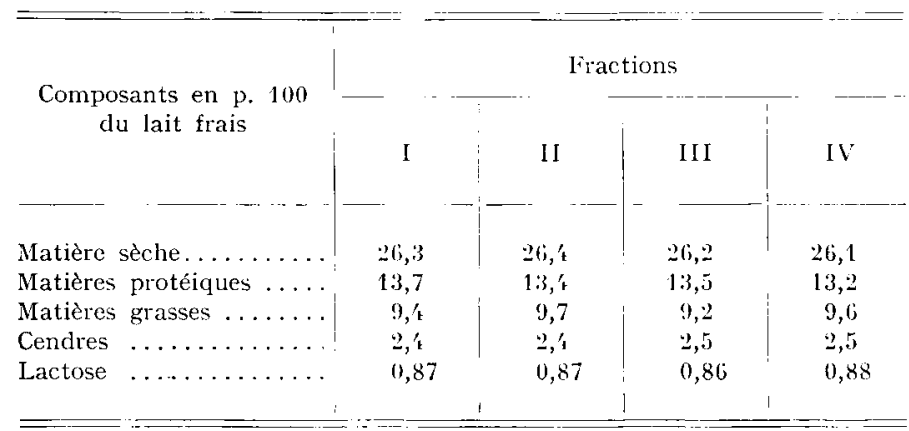

2. Evolution de la composition du lait au cours de la lactation

La composition chimique du lait moyen correspond aux données ci-dessous en grammes par $\mathrm{kg}$ de lait frais :

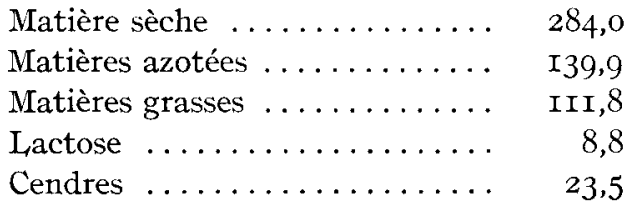


Nous avons donc un lait riche en protéines et en matières grasses, très pauvre en lactose.

Cette composition n'est pas stable au cours de la lactation, elle évolue principalement en fin de lactation, comme l'indique le tableau 3.

\section{TABLEAU 3}

Composition chimique du lait de lapine à divers stades de lactation

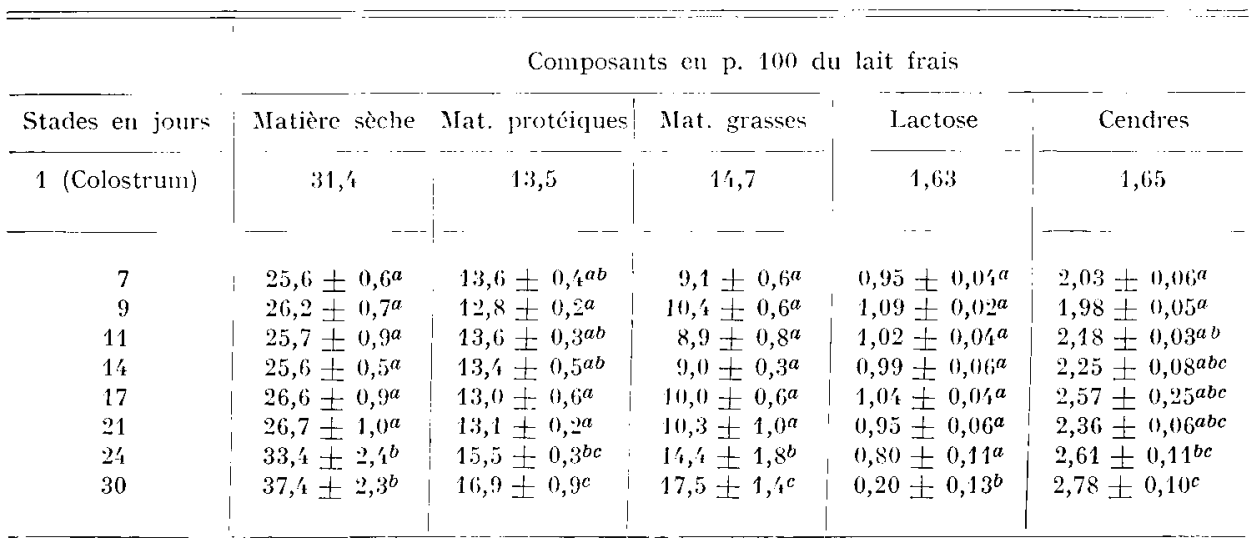

$a, b, c$ : Les nombres ayant en indice une lettre différente sont significativement différents au seuil $\mathrm{P}=0,05$.

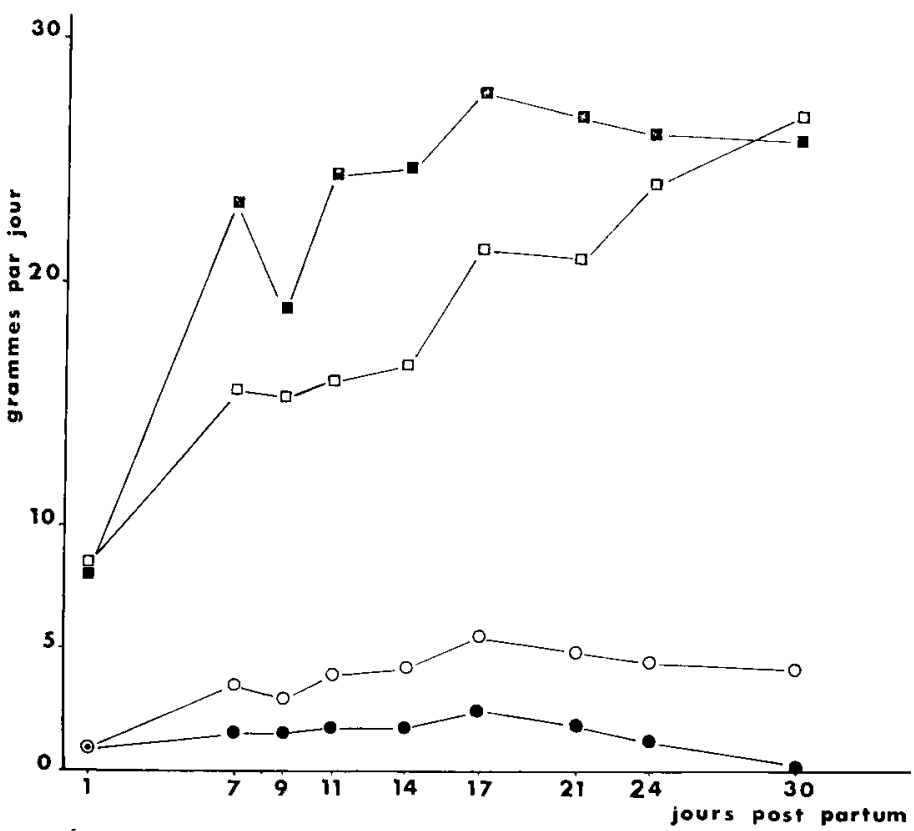

FIG. I. -- Evolution dans le temps des quantités totales d'éléments constitutifs du lait de lapine produites chaque jour

- - matières protéiques $\square-\square$ matières grasses

o - o matières minérales - lactose

(Moyenne de 6 échantillons par stade) 
Si le colostrum se distingue du lait moyen par sa teneur élevée en divers éléments sauf pour les cendres, la composition reste relativement stable ensuite jusqu'au $2 \mathrm{I}^{\mathrm{e}}$ jour. Dans le courant de la $4^{\mathrm{e}}$ semaine de lactation, on assiste d'une part à un enrichissement du lait en protéines, en matières grasses et en cendres, d'autre part, à la disparition presque complète du lactose. Ce dernier élément n'a d'ailleurs pas pu être mis en évidence sur 3 des six échantillons prélevés 30 jours après la mise bas. Il convient de remarquer que les lapines ayant donné ces 3 laits avaient une production laitière encore élevée au $30^{\mathrm{e}}$ jour.

La figure I donne l'évolution des quantités des divers composants du lait, exportées chaque jour. Les quantités de protéines exportées chaque jour augmentent jusqu'au $I 7^{e}$ jour et se stabilisent ensuite, il en va de même pour les cendres. Les matières grasses sont sécrétées en quantité croissante jusqu'au $30^{\mathrm{e}}$ jour, tandis que la production de lactose se réduit rapidement à partir $\mathrm{du} \mathrm{I} 7^{\mathrm{e}}$ jour.

\section{DISCUSSION}

\section{I. - Évolution de la composition du lait en cours de traite}

Les différents auteurs ayant analysé des échantillons de lait de lapine n'ont prélevé que de faibles échantillons de Io à $20 \mathrm{~g}$ (NEUmeister et KraUSE, I958; Coates et al., I964; Samperi et Celinino-Tosi, I966; Smith et al., ig68; Sampieri et al., I969; CowIE, I969). La question se pose donc de savoir si de tels prélèvements sont représentatifs du lait consommé par les lapereaux. L'absence de différence entre les 4 fractions d'un même échantillonnage laisse penser qu'un début de traite est suffisamment représentatif pour autoriser l'extrapolation de cette fraction à l'ensemble. Cependant, nos fractions représentent 25 p. Ioo du lait tété par les lapereaux, soit 50 à $60 \mathrm{~g}$ dans le cas de lapines en pleine lactation. Ces échantillons sont encore 2 à 5 fois plus importants que les prélèvements couramment pratiqués par les auteurs précités. En l'absence d'expérimentation plus avancée en la matière, nous pensons qu'il faut utiliser des échantillons représentant au moins $20 \mathrm{p}$. Ioo du lait normalement tété par les lapereaux, pour les études visant à déterminer la composition de l'ingété de ces derniers. Cette méthode nécessite la détermination préalable de la quantité à prélever de manière à connaître le degré de teprésentativité de l'échantillon obtent1.

La relative constance de la composition du lait au cours de la traite est en apparente opposition avec les résultats de Salmon-Legagneur (I965) sur la Truie, de LABussière (I969) chez la Brebis et de Johansson et al. (I952) chez la Vache. Cependant ces auteurs ont cherché à obtenir une traite complète de chaque femelle tandis que nous nous sommes limités à la quantité consommée par les lapereaux. A plusieurs occasions il nous a été donné de vérifier qu'une portéa de huit lapereaux ne prélève que 80 à $90 \mathrm{p}$. Ioo du lait présent dans la mamelle. Quelques traites de ces Io à $20 \mathrm{p}$. IOO restant nous ont montré qu'ils étaient beaucoup plus riches en matières grasses que les premiers $80 \mathrm{p}$. Ioo. D'autre part, nous avons utilisé des doses d'ocytocine 4 à Io fois plus élevées que celles libérées par la tétée d'une portée (Fuchs et WAGNER, I963). Il est donc possible que cette injection massive d'hormone post- 
hypophysaire ait entraîné un certain brassage du lait dans la mamelle, ne nous permettant pas d'enregistrer une éventuelle évolution. De même, nos arrêts pour changement de flacon, même relativement courts, ont pu modifier le mode d'excrétion normal du lait. En tout état de cause il ne nous a pas été possible de mettre en évidence d'évolution de la composition; si celle-ci existe malgré tout, comme pour les autres espèces domestiques, nous ne pensons pas qu'elle soit très importante car nous l'aurions alors mise, au moins partiellement, en évidence.

\section{2. - Evolution de la composition du lait au cours de la lactation}

A notre connaissance, la recherche d'une variation de la composition du lait de lapine au cours de la lactation n'a été tentée que par quelques auteurs. CoAtes et al. (I964) sur un nombre très limité de lapines Néozélandais blanc (NZW) ne trouvent pas de variation de la composition du lait entre la mise bas et la $3^{\mathrm{e}}$ semaine. CowIE, (I969) par contre, trouve comme nous, mais pour des lapines $N Z W$ et pour des lapines Hollandais, une évolution dans la composition du lait au cours de l'allaitement. Les variations pour les $N Z W$ sont samblables à ce que nous avons observées sur nos lapins Californien. Filles sont un peu moins marquées dans le cas des lapins Hollandais. Si les évolutions sont comparables, les niveaux des divers éléments sont différents.

Nous trouvons en effet un lait plus riche en protéines qu'en lipides à l'inverse des auteurs précités, seuls SAMPERI et CErLINO-TOSI (I966) mentionnent pour des lapines communes italiennes un lait à $\mathrm{I}_{4}, 2 \mathrm{p}$. Ioo de protéines et I0,5 - I I p. Ioo de lipides. De même, nous trouvons une teneur en lactose plus faible : I,o p. Ioo pour nos lapines Californien contre I,5 à 2,o p. Ioo pour les $N Z W$ de CoATes et al. (I964).

Pour le colostrum prélevé le lendemain de la mise bas, nous trouvons comme les auteurs précédents une forte teneur en matières sèches avec une prédominance des matières grasses, mais nous enregistrons une teneur plus forte en lactose que pour le lait à l'inverse de Cowie (Ig69).

L'utilisation de races différentes peut expliquer une partie des variations de composition des laits contrôlés par les auteurs. Il convient également de mentionner comme source de variation possible la technique de traite et le volume des échantillons recueillis. SAMPERI et CEII,INO-TOSI ( I966) et nous-mêmes mentionnons l'utiljsation d'une machine à traire tandis que tous les autres auteurs ont effectué une traite manuelle par massage plus ou moins prolongé de la mamelle. Nous pensons que ce massage a pu favoriser l'élimination préférentielle des matières grasses expliquant ainsi les teneurs élevées en lipides des laits ainsi obtenus. D'autre part, un échantillon de Io g de lait représentant 3 à 5 p. Ioo du lait présent nous semble difficilement représentatif de ce que les lapereaux peuvent consommer.

\section{CONCLUSION}

Au cours d'une même traite, la composition chimique du lait ne semble pas varier, tandis que la fraction restant dans la mamelle est plus riche en matières sèches et principalement en matières grasses. Un échantillon de 40 à 80 grammes de lait est donc susceptible de représenter valablement le lait consommé par les lapereaux. 
Au cours de la lactation, le lait passe d'un stade riche en matière sèche à dominante lipidique durant la phase colostrale pour se stabiliser à un taux un peu plus faible en matière sèche durant 3 semaines. Durant cette période, les laits obtenus par traite mécanique sont plus riches en protéines (I4 p. IOO) qu'en lipides (I I p. IOO). Dans le courant de la $4^{\text {e }}$ semaine, le lait s'enrichit en protéines, en lipides et en cendres tandis que le lactose a tendance à disparaître.

Reçu pour publication en février 1971.

\section{SUMMARY}

\section{THE CHEMICAL, COMPOSITION OF RABBIT'S MII, CHANGES DURING MILKING AND ACCORDING TO THE STAGE OF LACTATION}

Several samples of rabbit's milk were obtained at successive stages during the same mechanical milking period. The chemical composition does not seem to vary during a milking period representative of the amount of milk normally consumed by the litter, while the fraction remaining in the mammary gland is richer in dry matter and more particularly in lipids. However, a sample of milk to be used for analysis must represent at least a quarter of the total amount present.

During lactation, the composition of the milk is relatively stable until the end of the 3 rd week. The dry matter (28.8 p. I oo) is composed mainly of proteins (49 p. 100) and lipids (39 p. Ioo), while the smaller fractions are composed of lactose and ash ( $3 \mathrm{p}$. Ioo and $8 \mathrm{p}$. Ioo respectively). During the $4^{\text {th }}$ week, the milk becomes richer in proteins and, in particular, lipids, while the lactose tends to disappear.

\section{RÉFÉRENCES BIBLIOGRAPHIQUES}

Coates M. E., Gregory M. E.., Thompson S. Y., 1964. The composition of rabbit's milk. Brit. J. Nutr., 18, 563-586.

Cowle A. T., I969. Variations in the yield and composition of the milk during lactation in the rabbit and the galactopoietic effect of prolactin. J. Endocr., 44, 437-450.

Fichs A. R., WAGNeR ( $i$., I963. Quantitative aspects of release of oxytocin by suckling in unanesthestized rabbits. Acta endocr., 44, 581-592.

Jomansson I., Korkman N., Nelson N. J., I952. Studies on udder evacuation in dairy cows. I. The rise in fat percentage during milking. Acta A gric. Scand., 2, 43-8I.

Labussière J., r969. Importance, composition et signification des différentes fractions de lait obtenues successivement au cours de la traite mécanique des brebis. Ann. Zootech., 18, I85-I96.

Lebas F., r968. Mesure quantitative de la production laitière chez la Lapine. Ann. Zootech., 17, I69-I8z.

Llebas $F .$, I969. Alimentation lactée et croissance pondérale du lapin avant sevrage. Ann. Zootech., 13, $197-208$.

LFbas F., 1970. Description d'une machine à traire les lapines. Ann. Zootech., 19, 223-228.

Lebas F., Corring T., Courtot D., r97I. Équipement enzymatique du pancréas exocrine chez le Iapin, mise en place et ćvolution de la naissance au sevrage, relation avec la composition du régime alimentaire. Ann. Biol. anim. Bioch. Biophys., 11 (3) (sous presse).

Neumerster H., Krause B., 1956. Zum Nährstoffgehalt cler Kaninchenmilch. Arch. Gefligelz. $u$. Kleintierk., 5, I99-203.

Salmon-Legagneur F., I965. Quelques aspects des relations nutritionnelles entre la gestation et la lactation chez la Truie. Ann. Zootech., 14 (nº hors-série), I43 pp.

Samperi M., Cellino-Tosi A., ig66. La composizione del latte di conigila comportamento elettroforetico delle proteine sieriche a quadro acidico del lipidi. Conigli. Anim. Pelliccia, I968 (Io) 7 p.

Sampiert M., ValfRe F., Maletto S., ig69. Sulla componente acidico del grasso del latte di coniglia. Riv. Zootech. Agric. Vet., 7, I-9.

Sмiтh S., Watts R., Dils R., 1968. Quantitative gas-liquid chromatographic analysis of rodent milk triglycerides. J. Lip. Res, $\mathbf{9}, 52-57$. 